NBER WORKING PAPER SERIES

\title{
INTERGENERATIONAL HEALTH MOBILITY: MAGNITUDES AND IMPORTANCE OF SCHOOLS AND PLACE
}

\author{
Jason Fletcher \\ Katie M. Jajtner \\ Working Paper 26442 \\ http://www.nber.org/papers/w26442 \\ NATIONAL BUREAU OF ECONOMIC RESEARCH \\ 1050 Massachusetts Avenue \\ Cambridge, MA 02138 \\ November 2019
}

The authors would like to acknowledge Postdoctoral Trainee funding (K. Jajtner) under NIA grant T32 AG00129 and support from the Center for Demography of Health and Aging (CDHA) at the University of Wisconsin-Madison under NIA core grant P30 AG17266. Bhashkar Mazumder and participants at the University of Wisconsin - Madison Health Economics working group and Demography Seminar provided helpful suggestions for improvement. This research uses data from Add Health, a program project directed by Kathleen Mullan Harris and designed by J. Richard Udry, Peter S. Bearman, and Kathleen Mullan Harris at the University of North Carolina at Chapel Hill, and funded by grant P01-HD31921 from the Eunice Kennedy Shriver National Institute of Child Health and Human Development, with cooperative funding from 23 other federal agencies and foundations. Information on how to obtain the Add Health data files is available on the Add Health website (http://www.cpc.unc.edu/addhealth). No direct support was received from grant P01-HD31921 for this analysis. The views expressed herein are those of the authors and do not necessarily reflect the views of the National Bureau of Economic Research.

NBER working papers are circulated for discussion and comment purposes. They have not been peer-reviewed or been subject to the review by the NBER Board of Directors that accompanies official NBER publications.

(C) 2019 by Jason Fletcher and Katie M. Jajtner. All rights reserved. Short sections of text, not to exceed two paragraphs, may be quoted without explicit permission provided that full credit, including $\odot$ notice, is given to the source. 
Intergenerational Health Mobility: Magnitudes and Importance of Schools and Place Jason Fletcher and Katie M. Jajtner

NBER Working Paper No. 26442

November 2019

JEL No. I1,I12,I14,J62

\begin{abstract}
$\underline{\text { ABSTRACT }}$
Nascent research suggests intergenerational health mobility may be relatively high and nongenetic factors may make room for policy intervention. This project broadens this direction by considering heterogeneous intergenerational health mobility in spatial and contextual patterns. With 14,797 parent-child pairs from a school-based representative panel survey of adolescents (Add Health), this study finds large spatial variation in intergenerational health mobility in the United States. On average relative mobility in this sample is approximately 0.17 and expected health rank for children of parents at the 25th percentile of parent health is 47 . These metrics however mask substantial spatial heterogeneity. In cases of low health mobility, rank-rank slopes can approach 0.5 or expected child health rank may only be the 34 th percentile. Descriptive school- and contextual-level correlates of this spatial variation indicate localities with higher proportions of non-Hispanic blacks, school PTAs, or a school health education requirement may experience greater health mobility.
\end{abstract}

Jason Fletcher

University of Wisconsin-Madison

La Follette School of Public Affairs

1225 Observatory Drive

Madison, WI 53706

and NBER

jfletcher@lafollette.wisc.edu

Katie M. Jajtner

University of Wisconsin-Madison

Center for Demography of Health and Aging

1180 Observatory Dr. Rm. 4408

Madison, WI 53706

jajtner@wisc.edu 


\section{Introduction}

A growing literature on intergenerational relationships seeks to understand whether and why (or why not) the United States is truly a "land of opportunity". Examining the interconnectedness of generations is important not only to understand present disparities in key socioeconomic outcomes, but also in planning for future generations and identifying potential interventions. This study undertakes the examination of intergenerational health mobility, a branch of the intergenerational persistence literature which often examines the intergenerational persistence of socioeconomic status as defined by income or earnings. Although health is often neglected in research on intergenerational mobility, there is a small but growing body of literature to explicitly examine intergenerational health mobility.

Health throughout the life course is pivotal to many experiences in life including education, labor market participation, and aging. Poor health in childhood is associated with lower educational attainment (Case, Fertig, \& Paxson 2005; Haas \& Fosse 2008), which in turn is associated with lower likelihood of successful aging and higher mortality (McLaughlin 2017; Meara, Richards, \& Cutler 2008). Self-rated general health status itself may be a predictor of mortality (DeSalvo, Bloser, Reynolds, He, \& Muntner 2006; Idler \& Angel 1990). Experiencing poor health during prime working ages can also have lasting effects. Healthy men on average are found to work more, earn more, and have higher net worth (Hokayem \& Ziliak 2014). In the event that ill health prevents an individual from actively or fully engaging in the labor market, such work limitations are associated with significant drops in earnings as well as post tax-transfer income up to a decade after onset of a work-limiting health condition (Meyer \& Mok 2018).

Understanding the intergenerational persistence of health is warranted for a variety of reasons. First, it is important to determine whether, and how much, of an individual's health trajectory is potentially modifiable. That is to say that if health is perfectly transmitted across generations, then there could be little room for policy to moderate outcomes and such efforts may be a waste. However, under the more likely assumption that there are some environmental components to health, then an intergenerational approach can help illuminate expected future outcomes for subsequent generations. Third, the approach could identify possible spillovers of interventions that may simultaneously benefit multiple generations. Finally, as research continues to develop, optimal timing of interventions to maximize spillovers and improve health may be feasible with 
a solid understanding of the intergenerational transmission of health. Ultimately, such improvements in general health if successful could theoretically lead to reduced public health burden in terms of lower medical expenses. If better health translates into improved work capacity (and correspondingly less work-limiting health conditions), social costs for programs such as Social Security Disability Insurance, which constitutes a major and growing public expenditure (Autor \& Duggan 2006), may be alleviated.

Results from this study suggest there is a non-negligible intergenerational persistence in health that is present for both biological and adoptee parent-child pairs. While these nationally representative estimates are informative to gauge overall health persistence, the metrics mask significant heterogeneity in the United States, and characteristics of geographic localities are used to explore correlations with health mobility. Areas that are suburban or rural tend to experience less relative mobility, while localities with higher income inequality may have higher health mobility on average. Granular school-based characteristics such as whether a school has a Parent-Teacher Association or a Health Education requirement are found to be positively associated with intergenerational health mobility.

The remainder of this paper is organized into four additional sections. The second section presents the relevant literature to form the basis of the current analysis. The third section discusses the methods and data, and the fourth section presents the main results of the analysis. The final section discusses results and implications.

\section{Background}

Previous research in intergenerational mobility typically examines the persistence of socioeconomic status as measured by income, earnings, or educational attainment. This literature has uncovered that contrary to the notion of the "American Dream", where anyone can achieve economic success if he/she is willing to work for it, the United States tends to experience relatively lower levels of intergenerational economic mobility relative to peer income nations (Corak 2013; Solon 2002). In addition to these low national estimates, scholarship has shown broad-based underlying heterogeneity in terms of race/ethnicity (Chetty, Hendren, Jones, \& 
Porter 2018; Hertz 2005; Mazumder 2014) and geography (Chetty, Hendren, Kline, \& Saez 2014).

A much smaller literature explores intergenerational persistence of health. An early example examined the intergenerational correlation of birthweight in California, finding low birthweight mothers are more likely to have low birthweight children, and the intergenerational persistence of birthweight was around 0.2 (Currie \& Moretti 2007). Using outcomes a little later in the life course, Thompson (2014) examines the intergenerational transmission of asthma, hay fever, chronic headaches (migraines), and diabetes, finding a range of estimates from 0.006 for diabetes to 0.276 for hay fever (Thompson 2014). The intergenerational elasticity of body mass index has been estimated between 0.3-0.4 (Classen 2010; Classen \& Thompson 2016). An example examining the intergenerational persistence of mental health in Britain also found a correlation of 0.16 (Johnston, Schurer, \& Shields 2013). What emerges from this literature is a relatively consistent estimate of intergenerational health persistence in the $0.2-0.3$ range.

A recent paper that is most similar to the present analysis uses the Panel Study of Income Dynamics (PSID) to examine intergenerational health mobility in self-reported health status, corroborating previous estimates of intergenerational health persistence (Halliday et al. 2018). Notably the authors also are the first to apply the concept of "absolute mobility" from Chetty et al. (2014) to the health context. This is distinguished from "relative mobility" - the slope coefficient of a standard OLS regression (intergenerational elasticity, or when adjusted by respective standard deviations of the variable in each generation, intergenerational correlation). This paper is also the first to apply the rank-based approach from Chetty et al. (2014), which has been found to also exhibit improved properties regarding the standard assumption of linearity for estimation in the health context (Wong, Mazumder, \& Halliday 2019).

While Halliday et al. (2018) make important contributions to examining the intergenerational persistence of general health status, the dataset limits the ability to explore spatial variation in mobility. The PSID affords many advantages including regular observations of individuals over time, a genealogical design, up to 50 years of data on multiple generations, and in recent years, the inclusion of rich data on health conditions. However, the sample size can be relatively small, does not capture a large Hispanic population, and it does not afford the opportunity to examine spatial variation in intergenerational health mobility, which is likely to exist in the United States 
given documented heterogeneity in intergenerational economic mobility (Chetty et al. 2014). Using the National Longitudinal Study of Adolescent to Adult Health (Add Health), the present paper complements and extends previous work in two important ways. First, the sample size is more than $80 \%$ larger, allowing corroboration of important estimates including race/ethnicity stratifications in addition to specifically examining Hispanics separately. The larger sample also yields a sizable subsample of adoptees which will assist in determining the potential role for nongenetic factors, or public policy to moderate intergenerational transmission, complementing previous work regarding the genetic role for intergenerational health persistence (Classen \& Thompson 2016; Thompson 2014). Second, a school-based sample design in addition to the larger sample allows for examining spatial variation of mobility estimates.

This study addresses four key research questions. First, it estimates intergenerational health mobility using a large nationally representative sample. Following previous literature, it also estimates the expected rank in a national health distribution for a child conditional on his/her parent(s) health status. Third, by stratifying key mobility metrics for biological and adoptee parent-child pairs, this study considers whether the environment (or non-genetic factors) play a role in observed intergenerational health mobility. Finally, the heterogeneity of mobility metrics by race/ethnicity, parent and child characteristics, and geography is explored.

\section{Methods and Data}

Intergenerational health mobility is estimated following traditional specifications in the literature of intergenerational economic mobility (Mazumder 2005; Solon 1992, 2004), with recent applications to intergenerational health mobility (Akbulut-Yuksel \& Kugler 2016; Classen 2010; Currie \& Moretti 2007; Halliday et al. 2018; Johnston et al. 2013). Specifically, parent’s selfreported health status $\left(S R H S_{p}\right)$ is regressed on child's self-reported health status $\left(S R H S_{c}\right)$ using Ordinary Least Squares controlling for age in quadratic form in both generations $\left(C_{z}\right)$ as in equation (1).

$$
S R H S_{c}=\beta_{0}+\beta_{1} S R H S_{p}+\sum \beta_{z} C_{z}+\varepsilon
$$


Two metrics of intergenerational mobility are measured from equation (1). The first is referred to as "relative" mobility in some literature (Chetty et al. 2014; Halliday et al. 2018), and is measured by $\beta_{1}$. The second establishes the expected outcome for children conditional on a parent's health status - what Chetty et al. (2014) conceptualize as "absolute” mobility - is measured as in equation (2).

$$
E\left(S R H S_{c} \mid S R H S_{p}=x\right)=\beta_{0}+\beta_{1}(x)
$$

Self-rated health status is typically measured on a five-point Likert scale with one representing poor health and five representing excellent health. As the marginal distribution of the Likert scale for self-rated health is likely non-constant, this study adopts a latent variable approach such that self-rated health responses represent an underlying, but unobserved, health status. The HALex index maps self-rated health status to unobserved latent health conceptualizing perfect health with a score of one and death with a score of zero (Erickson 1998; Erickson, Wilson, \& Shannon 1995), and has been applied previously in the literature to address concerns surrounding the marginal distribution of health from the Likert scale (Halliday et al. 2018; Johnson \& Schoeni 2011). Following these examples, this analysis assigns the midpoint of established HALex interval values of $15,50,77.5,90$, and 97.5 to self-rated health statuses of poor, fair, good, very good, and excellent in both generations.

Measuring the intergenerational transmission of health using an OLS specification requires an assumption of linearity. Research in intergenerational economic mobility suggests using logged observed income or earnings may present some non-linearities that are typically resolved using a rank-based approach (Chetty et al. 2014). Analogously, a recent working paper suggests that estimates of intergenerational health mobility, the Intergenerational Health Association (IHA) from Halliday et al. (2018), which use simply the HALex adjusted levels for self-rated health status in both generations may produce estimates that are biased downward by $10-20$ percent. However, ranking health statuses in each generation and then applying these rank-based measures to equation (1) does not appear to yield any meaningful bias to estimates when adopting the assumption of linearity for OLS (Wong et al. 2019). 
The preferred results in this analysis therefore adopt a rank-based measure of self-rated health from the HALex adjusted scale, although both formulations are estimated. Most estimates that follow will incorporate four main metrics: (1) the IHA slope, $\beta_{1}$ coefficient from equation 1 using HALex level self-rated health in both generations, (2) a Rank slope, $\beta_{1}$ coefficient from equation 1 using rank-based self-rated health in both generations, (3) the expected health rank of a child conditional on his/her parent having health rank at the $25^{\text {th }}$ and (4) $75^{\text {th }}$ percentile of parent health from equation 2. The first two are measures of relative mobility, where more (less) mobility is characterized by a lower (higher) metric. The latter two estimates are used following literature selecting these percentiles (Chetty et al. 2014; Halliday et al. 2018) to gauge absolute mobility with the understanding that if mobility were perfect the expected health rank for all children regardless of parents' health rank would be the median.

Data comes from The National Longitudinal Study of Adolescent to Adult Health (Add Health), a school-based nationally representative panel survey of adolescents from grades 7-12. This study incorporates child reports of self-rated health status from four waves of data (1994-1995, 1996, 2001-2002, and 2008-2009) and parents' self-reported health from the first wave from a total of 131 schools. The school-based design is critical for this analysis, as it allows for examining spatial variation in estimates, which has not previously been explored in the intergenerational health mobility literature. The final full sample consists of 14,797 parent-child pairs.

Various stratifications of equation 1 help understand the heterogeneity of intergenerational health mobility that exists in the United States. In particular, this analysis examines such heterogeneity based on a variety of factors found to be influential in the literature on intergenerational economic mobility such as race/ethnicity (Chetty et al. 2018; Hertz 2005; Mazumder 2014), and geography (Chetty et al. 2014). Additionally, we incorporate stratified specifications to examine other factors associated with possible disadvantage including health insurance (as in Halliday et al. 2018), parent marital status, and parent education. These results are weighted to reflect more nationally-representative metrics. Spatial variation in particular is examined further using Census-tract specific characteristics to examine whether there are certain characteristics (demographic, socioeconomic, and/or school-specific programs) which may correlate with observed intergenerational health mobility measured at the school level. 


\section{Results}

For our nationally representative sample of the United States, relative intergenerational health mobility as measured by a rank-rank slope is estimated at 0.174 (95\% confidence interval: 0.141 -0.206 ). Children whose parents are in relatively poorer health (at the $25^{\text {th }}$ percentile of parent health) are anticipated to reach near the $47^{\text {th }}$ percentile of health on average, while children whose parents are in relatively better health (the $75^{\text {th }}$ percentile of parent health rank) are expected to achieve near the $56^{\text {th }}$ percentile of health as adults. Estimates using the PSID suggest the rank-rank slope coefficient to be 0.261 and expected child health rank for those whose parents are at the $25^{\text {th }}\left(75^{\text {th }}\right)$ percentile of parent health is 44.3 (57.4) (Halliday et al. 2018). Within these nationally representative estimates however is significant heterogeneity by race/ethnicity, parent and child characteristics, and geography. Results also point to non-genetic factors exerting some influence on intergenerational health mobility as adoptive parent-child pairs also experience non-zero relative mobility.

Heterogeneity in intergenerational health mobility is present by race/ethnicity (Figure 1 and Table A1 in the Appendix). Specifically, non-Hispanic black and Hispanic parent-child pairs may experience greater relative mobility, meaning these subpopulations observe lower slope estimates both in terms of level health and health rank, relative to non-Hispanic whites. Figure 1 however suggests only Hispanic parent-child pairs have statistically more relative mobility. Children born to parents in relatively poorer health (at the $25^{\text {th }}$ percentile of parent health) are generally not expected to reach median health as adults, and point estimates are generally consistent across race-ethnicity categories. Hispanic children though may have the best chances of achieving median health as adults, but differences do not appear statistically different. Meanwhile children born to parents in relatively good health (the $75^{\text {th }}$ percentile of parent health rank) exhibit a bit more heterogeneity in terms of expected child rank. Estimates suggest nonHispanic black children and Hispanic children may experience more mean reversion, landing nearer the $50^{\text {th }}$ percentile of health rank relative to their non-Hispanic white counterparts. Coefficient results are somewhat in contrast to results from Halliday et al. (2018), who find black parent-child pairs experience a coefficient of relative mobility approximately half that of their white counterparts as well as observing a ten percentile gap in expected health rank for children whose parents are at the $25^{\text {th }}$ percentile of parent health rank. 
Figure 1: Intergenerational health mobility stratified by race-ethnicity
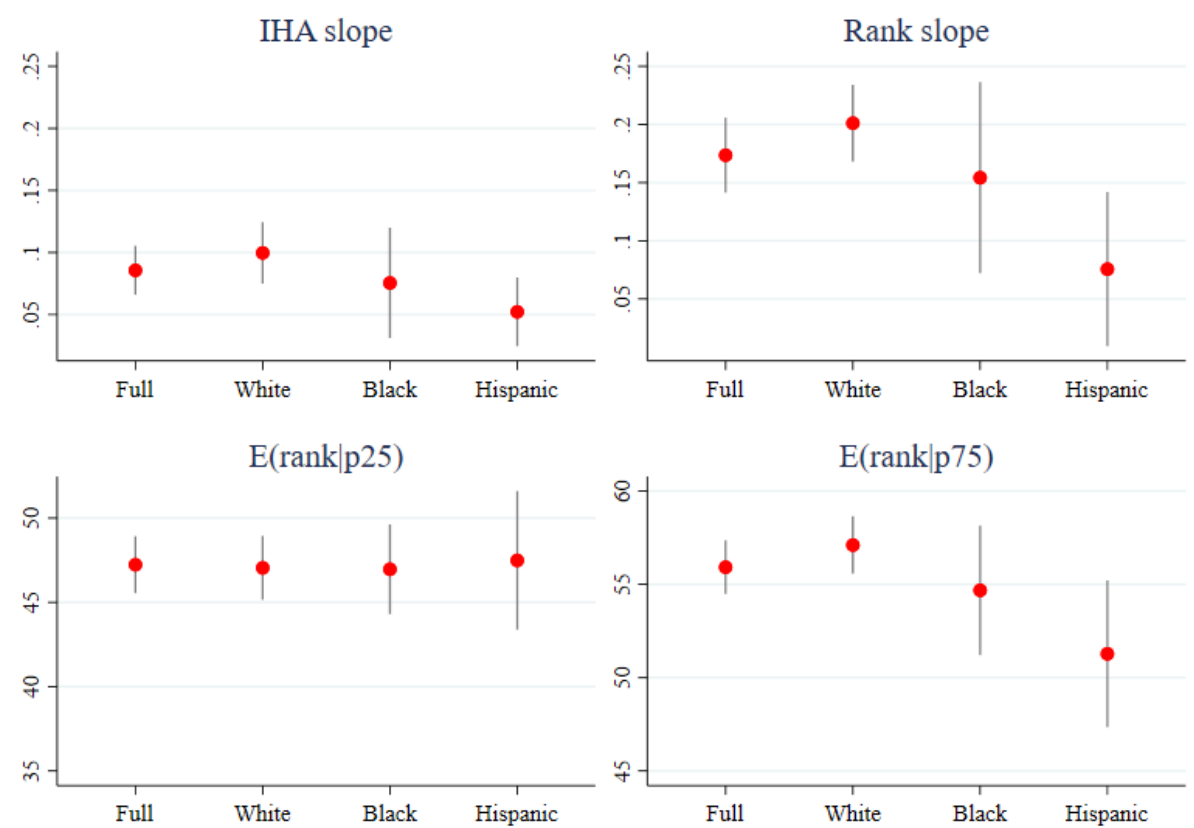

Source: Authors' calculations using Add Health

Notes: IHA slope is the Intergenerational Health Association slope ( $\beta_{1}$ coefficient from equation 1 ) using HALex level self-rated health in both generations. Rank slope is the $\beta_{1}$ coefficient using health ranks in both generations. $\mathrm{E}$ (rank|p25) is the expected health rank for a child conditional on his/her parent observed at the $25^{\text {th }}$ percentile of parent health, and $\mathrm{E}(\mathrm{rank} \mid \mathrm{p} 75)$ is the expected health rank for children whose parent(s) are observed at the $75^{\text {th }}$ percentile of parent health. Estimates are weighted, and use the full Add Health sample. Red dots represent point estimates and vertical lines are the corresponding 95\% confidence intervals.

Certain characteristics of parent-child pairs can be identified as disadvantageous in Table 1. For example, children who ever were without health insurance as young adults (in wave four) generally observe lower expected health ranks relative to those who always had health insurance, although disadvantage appears concentrated in non-Hispanic white populations and to a limited degree among non-Hispanic blacks at lower parent health ranks (gaps are not statistically significant, but in the hypothesized direction). Halliday et al. (2018) also estimate lower expected child health rank for children exposed to disadvantageous environments with respect to health insurance. Children whose parent(s) were unmarried or whose mothers had less than a High School education similarly tend to observe lower expected health relative to children of married parents and mothers with more than a High School credential. The gap observed between "advantaged" and "disadvantaged" parent-child pairs though is not constant throughout the parent health distribution, or by race/ethnicity. Generally speaking, the gap in each of these 
characteristics increases as parent health increases, which reflects a typically flatter slope for “disadvantaged” parent-child pairs (signaling higher relative mobility) or that such characteristics may matter more when the parent is in better health. However, while nonHispanic white parent-child pairs observe an increasing gap over parent health rank with respect to parent marital status, the gradient appears much stronger for Hispanics, and inverted for nonHispanic blacks.

Table 1: Expected absolute mobility conditional on parent health rank \& personal characteristics

\begin{tabular}{|c|c|c|c|c|c|c|c|c|c|c|c|c|}
\hline \multicolumn{13}{|c|}{ Mother's Educational Attainment } \\
\hline & \multicolumn{3}{|c|}{ Full Sample } & \multicolumn{3}{|c|}{ non-Hispanic white } & \multicolumn{3}{|c|}{$\underline{\text { non-Hispanic black }}$} & \multicolumn{3}{|c|}{ Hispanic } \\
\hline & A & $\mathrm{D}$ & Gap & A & $\mathrm{D}$ & Gap & A & $\mathrm{D}$ & Gap & A & $\mathrm{D}$ & Gap \\
\hline $10^{\text {th }}$ & 45.8 & 44.7 & 1.1 & 47.3 & 36.8 & $10.5^{* *}$ & 44.7 & 38.9 & 5.8 & 44.2 & 51.2 & $-7+$ \\
\hline $25^{\text {th }}$ & 48.9 & 44.6 & $4.3^{*}$ & 50.2 & 38.6 & $11.6^{* * *}$ & 47.4 & 39.1 & $8.3^{*}$ & 47.8 & 49.7 & -1.9 \\
\hline $50^{\text {th }}$ & 54.2 & 44.4 & $9.7^{* * *}$ & 55.2 & 41.6 & $13.5^{* * *}$ & 51.8 & 39.4 & $12.4^{*}$ & 53.7 & 47.2 & $6.5^{*}$ \\
\hline $75^{\text {th }}$ & 59.4 & 44.3 & $15.1^{* * *}$ & 60.1 & 44.6 & $15.5^{* * *}$ & 56.2 & 39.6 & $16.5^{*}$ & 59.6 & 44.7 & $14.9 * * *$ \\
\hline $90^{\text {th }}$ & 62.6 & 44.2 & $18.4^{* * *}$ & 63 & 46.4 & $16.6^{* *}$ & 58.8 & 39.8 & $19 *$ & 63.1 & 43.2 & $20 * * *$ \\
\hline
\end{tabular}

Parent Marital Status

\begin{tabular}{|c|c|c|c|c|c|c|c|c|c|c|c|c|}
\hline & \multicolumn{3}{|c|}{$\underline{\text { Full Sample }}$} & \multicolumn{3}{|c|}{ non-Hispanic white } & \multicolumn{3}{|c|}{ non-Hispanic black } & \multicolumn{3}{|c|}{$\underline{\text { Hispanic }}$} \\
\hline & A & $\mathrm{D}$ & Gap & A & $\mathrm{D}$ & Gap & A & $\mathrm{D}$ & Gap & A & $\mathrm{D}$ & Gap \\
\hline $10^{\text {th }}$ & 45.5 & 42.8 & 2.7 & 44 & 44.4 & -0.4 & 50.6 & 39.3 & $11.3^{* * *}$ & 47.9 & 42.9 & 5.0 \\
\hline $25^{\text {th }}$ & 48.2 & 44.7 & $3.5^{+}$ & 47.1 & 46.7 & 0.5 & 51.7 & 42.3 & $9.4^{* * *}$ & 49.6 & 42.7 & $6.9 *$ \\
\hline $50^{\text {th }}$ & 52.8 & 47.9 & $4.9 * *$ & 52.4 & 50.4 & 2.0 & 53.5 & 47.4 & $6.1^{*}$ & 52.4 & 42.3 & $10^{* *}$ \\
\hline $75^{\text {th }}$ & 57.4 & 51 & $6.3^{* * *}$ & 57.7 & 54.1 & 3.6 & 55.3 & 52.4 & 2.9 & 55.2 & 42 & $13.2^{* *}$ \\
\hline $90^{\text {th }}$ & 60.1 & 53 & $7.1^{* * *}$ & 60.8 & 56.3 & $4.5^{+}$ & 56.4 & 55.4 & 1.0 & 56.9 & 41.8 & $15.1^{* *}$ \\
\hline
\end{tabular}

Child Health Insurance Status

\begin{tabular}{|c|c|c|c|c|c|c|c|c|c|c|c|c|}
\hline & \multicolumn{3}{|c|}{$\underline{\text { Full Sample }}$} & \multicolumn{3}{|c|}{$\underline{\text { non-Hispanic white }}$} & \multicolumn{3}{|c|}{ non-Hispanic black } & \multicolumn{3}{|c|}{$\underline{\text { Hispanic }}$} \\
\hline & A & $\mathrm{D}$ & Gap & A & $\mathrm{D}$ & Gap & A & $\mathrm{D}$ & Gap & A & $\mathrm{D}$ & Gap \\
\hline $10^{\text {th }}$ & 46.3 & 40.9 & $5.5^{* *}$ & 46.4 & 39.2 & $7.3^{* *}$ & 46.1 & 39.7 & 6.4 & 47.1 & 45.8 & 1.3 \\
\hline $25^{\text {th }}$ & 49.2 & 42.8 & $6.5^{* * *}$ & 49.7 & 41.1 & $8.7 * * *$ & 47.9 & 43.4 & 4.6 & 48.4 & 46.9 & 1.6 \\
\hline $50^{\text {th }}$ & 54.1 & 45.9 & $8.2 * * *$ & 55.2 & 44.3 & $11^{* * *}$ & 51 & 49.5 & 1.5 & 50.7 & 48.6 & 2.1 \\
\hline $75^{\text {th }}$ & 58.9 & 49.1 & $9.9 * * *$ & 60.8 & 47.4 & $13.3^{* * *}$ & 54 & 55.6 & -1.5 & 52.9 & 50.4 & 2.6 \\
\hline $90^{\text {th }}$ & 61.8 & 50.9 & $10.9 * * *$ & 64.1 & 49.4 & $14.7^{* * *}$ & 55.9 & 59.2 & -3.4 & 54.3 & 51.4 & 2.9 \\
\hline
\end{tabular}

Source: Authors' calculations using Add Health

Notes: A represents the "advantaged" group (i.e. Mothers with more than a High School credential, married parents, or a child always with health insurance). D represents the "disadvantaged" group (i.e. Mothers with less than High School, unmarried parents, or a child who was ever uninsured). The Gap is the absolute difference in expected rank conditional on parent rank: ${ }^{* * *} p<0.001,{ }^{* *} p<0.01,{ }^{*} p<0.05,+p<0.1$ 
One particular advantage to Add Health is the ability to observe a sufficient number of adoptive parent-child pairs relative to biological parent-child pairs to better understand the potential role genetics may play in the intergenerational transmission of health. For this exercise, the main focus is on the $\beta_{1}$ coefficients from equation 1 , or relative mobility, to get at the intergenerational transmission. Results from this analysis suggest that while intergenerational transmission is certainly stronger for biological parent-child pairs, reflecting both genetic and environment factors, intergenerational transmission due to the environment is non-negligible with a statistically significant IHA slope estimate of 0.060 and rank slope estimate of 0.117 , representing a 39\% and 32\% reduction in slope estimates from the biological parent-child pair sample respectively. ${ }^{1}$

National-based estimates for intergenerational health mobility also mask underlying heterogeneity in geography. Figure 2 (and Table A2 in the Appendix) demonstrate the stark differences in all four mobility metrics when measured at the school level. Regardless of the metric considered, some schools experience high mobility while some experience low mobility. In some cases of particularly low mobility, rank slope estimates can approach 0.5 while other places experience rank slopes near zero. Similarly, in some locations, children born to parents in relatively poor health (at the $25^{\text {th }}$ percentile of parent health) can expect only a nine-percentile improvement over his/her parents' health rank while in other locations children born to parents experiencing such health may achieve greater than median health as adults. Meanwhile children born to parents in good health (at the $75^{\text {th }}$ percentile) may be expected to maintain a similar health status as adults for some schools while they could also be expected to experience health that is below the median as adults in other locations.

\footnotetext{
${ }^{1}$ This exercise does not make use of the Add Health weights or complex survey design. Adopted children were part of a genetic oversample in Add Health and applying weights dramatically reduces sample size in this subpopulation. Biological parent-child pair subsamples however remain robust to estimation with or without weights.
} 
Figure 2: School-stratified estimates of intergenerational health mobility
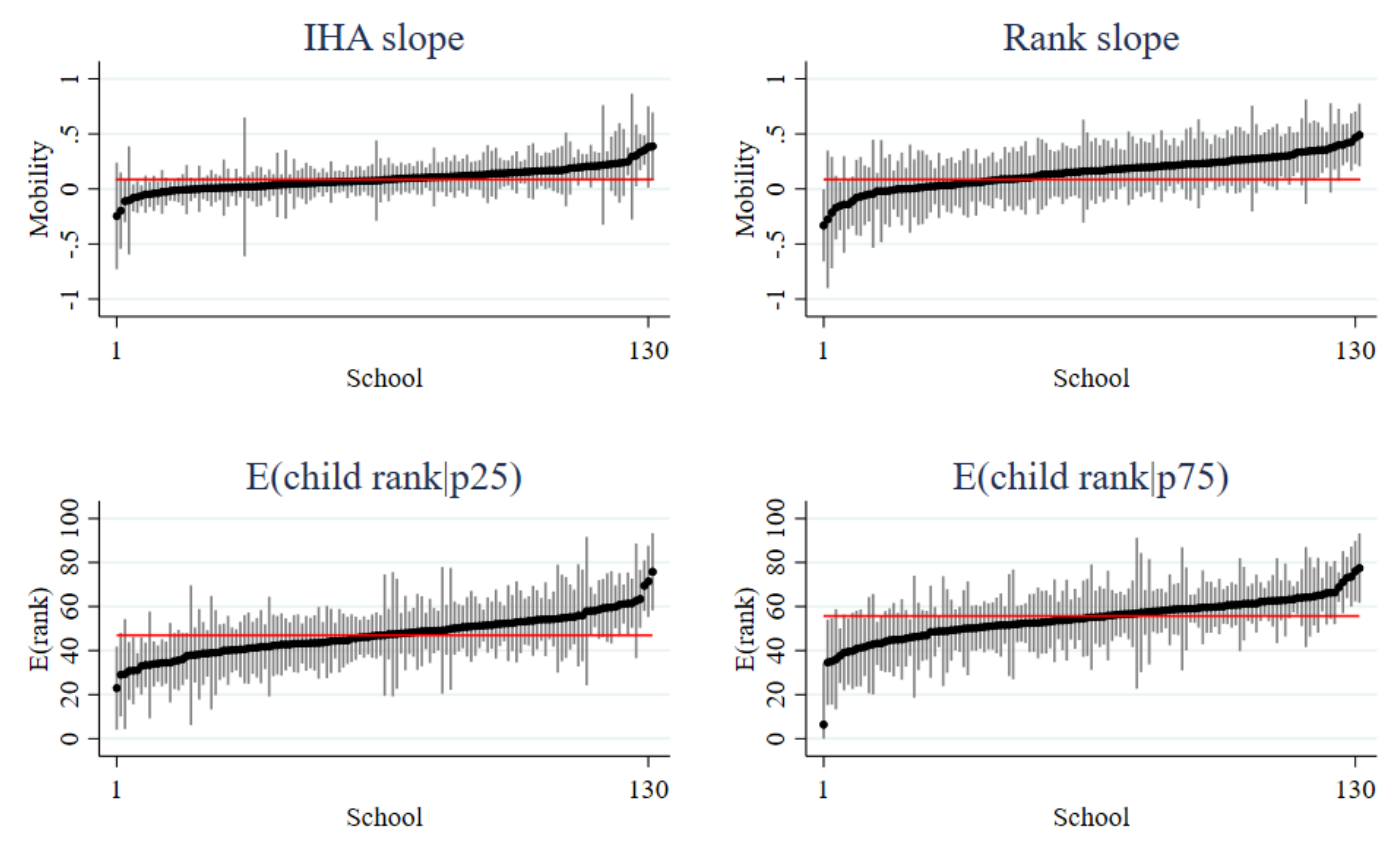

- Estimate

Full Sample

Core children only from eligible schools

Source: Authors' calculations using Add Health

Notes: IHA slope is the Intergenerational Health Association slope ( $\beta_{1}$ coefficient from equation 1 ) using HALex level self-rated health in both generations. Rank slope is the $\beta_{1}$ coefficient using health ranks in both generations. $\mathrm{E}(\mathrm{rank} \mid \mathrm{p} 25)$ is the expected health rank for a child conditional on his/her parent observed at the $25^{\text {th }}$ percentile of parent health, and $\mathrm{E}(\mathrm{rank} \mid \mathrm{p} 75)$ is the expected health rank for children whose parent(s) are observed at the $75^{\text {th }}$ percentile of parent health. Estimates are not weighted, but use only children identified in the core sample to produce school-specific results. Red lines represent the full (national) estimates, while black dots/line represent various school-specific estimates. Vertical lines are the corresponding 95\% confidence intervals for individual school estimate.

Following Chetty et al. (2014), we also explore contextual correlates of our school-based mobility estimates. These place-specific correlates of intergenerational health mobility appear in Table 2. Rural and suburban areas tend to experience less intergenerational health mobility in terms of relative rank slope and absolute mobility, although this is only statistically significant at the $10 \%$ level. Point estimates however also suggest rural areas on average could have lower absolute mobility as well. Suburban children may also experience less mobility, but the magnitudes tend to be lower with the exception of the IHA slope. Race and ethnicity characteristics of the area also seem to matter for health mobility. Areas with higher minority populations tend to experience higher relative mobility (consistent with results from raceethnicity stratified estimation), but these estimates also identify a statistically lower expected 
rank for children born to parents in good health (the $75^{\text {th }}$ percentile) in areas with higher minority populations. A larger portion of single parents in the area is associated with increased relative health mobility (IHA and rank slopes are lower), while there does not appear to be a correlation with absolute upward mobility. The area's socioeconomic characteristics may also matter; however, average socioeconomic status of the area (as measured by median income) does not have a robust relationship with any of these mobility metrics. Point estimates suggest there may be lower health mobility across all metrics in areas with higher median income, but estimates are not statistically significant. Income inequality on the other hand (as measured by the standard deviation in income for the area) may make a difference, where areas with high income inequality tend to experience greater health mobility; however, only the IHA slope is statistically significant $^{2}$. Finally, there are specific school characteristics that are associated with health mobility. Schools with a Parent-Teacher Association have lower rank slopes (higher relative mobility), while schools with a health education requirement may observe relatively higher health rank (nearly a five percentile bump) for their students whose parents are at the $25^{\text {th }}$ percentile of parent health.

Table 2: Correlates of intergenerational health mobility

\begin{tabular}{|c|c|c|c|c|c|}
\hline & & $\begin{array}{l}\underline{\text { IHA }} \\
\text { slope }\end{array}$ & $\begin{array}{l}\underline{\text { Rank }} \\
\underline{\text { Slope }}\end{array}$ & $\underline{E(r a n k \mid p 25)}$ & $\underline{E(\operatorname{rank} \mid p 75)}$ \\
\hline & Full Sample Estimate: & 0.086 & 0.174 & 0.472 & 0.559 \\
\hline \multirow{4}{*}{1} & Rural & 0.029 & $0.078^{+}$ & -0.041 & -0.002 \\
\hline & & $(0.029)$ & $(0.042)$ & $(0.025)$ & $(0.026)$ \\
\hline & Suburban & $0.039+$ & 0.044 & -0.023 & -0.001 \\
\hline & & $(0.020)$ & $(0.030)$ & $(0.018)$ & $(0.019)$ \\
\hline \multirow{6}{*}{2} & \% non-Hispanic black & -0.037 & $-0.146 * *$ & -0.018 & $-0.091 * *$ \\
\hline & & $(0.036)$ & $(0.051)$ & $(0.031)$ & $(0.032)$ \\
\hline & \% Hispanic & -0.050 & -0.092 & -0.050 & $-0.097+$ \\
\hline & & $(0.061)$ & $(0.087)$ & $(0.054)$ & $(0.054)$ \\
\hline & \% Other race & -0.197 & -0.153 & 0.081 & 0.004 \\
\hline & & $(0.126)$ & $(0.179)$ & $(0.110)$ & $(0.112)$ \\
\hline \multirow{2}{*}{3} & \% Single Parents & $-0.006 *$ & $-0.012 * *$ & 0.003 & -0.003 \\
\hline & & $(0.003)$ & $(0.004)$ & $(0.002)$ & $(0.003)$ \\
\hline
\end{tabular}

\footnotetext{
${ }^{2}$ To contextualize the coefficient on standard deviation in income, it ranges from approximately 20 to 50 with an interquartile range of 25 to 32 .
} 


\begin{tabular}{clcccc} 
& Median Income & 0.002 & 0.002 & -0.002 & -0.000 \\
\multirow{2}{*}{4} & & $(0.002)$ & $(0.004)$ & $(0.002)$ & $(0.002)$ \\
& \multirow{2}{*}{$\sigma$ Income } & $-0.007^{*}$ & -0.007 & 0.003 & -0.001 \\
& & $(0.003)$ & $(0.005)$ & $(0.003)$ & $(0.003)$ \\
\hline \multirow{2}{*}{5} & \multirow{2}{*}{ School PTA } & -0.008 & $-0.097^{*}$ & 0.034 & -0.014 \\
& & $(0.034)$ & $(0.048)$ & $(0.029)$ & $(0.031)$ \\
\hline \multirow{2}{*}{6} & \multirow{2}{*}{ Health Educ Required } & -0.013 & -0.013 & $0.049+$ & 0.042 \\
& & $(0.032)$ & $(0.047)$ & $(0.028)$ & $(0.029)$ \\
\hline
\end{tabular}

Source: Authors' calculations using Add Health

Notes: All models control for regional fixed effects, models in rows $2-4$ additionally control for urbanicity (row 1 ). Standard errors in parentheses, ${ }^{* *} \mathrm{p}<0.01,{ }^{*} \mathrm{p}<0.05,+\mathrm{p}<0.1$

\section{Discussion}

A nationally representative school-based sample of adolescents in the United States is used to estimate intergenerational health mobility in relative and absolute terms. Relative mobility using health ranks in both generations is found to be 0.174 (95\% confidence interval: $0.141-0.206$ ), under the assumption of linearity in the measure (Halliday et al. 2018; Wong et al. 2019). Absolute mobility for children whose parents are at the $25^{\text {th }}\left(75^{\text {th }}\right)$ percentile of parent health is estimated to be 47.2 (55.9), meaning children are expected to reach approximately the $47^{\text {th }}$ and $56^{\text {th }}$ percentiles respectively conditional on parent health rank. These nationally representative estimates however mask significant heterogeneity in the United States. Specifically, this study uncovers heterogeneous health mobility based on parent and child characteristics, geography, and school characteristics. Results also broadly suggest that policies may influence intergenerational health mobility. Not only are school characteristics such as having a PTA or health education requirement found to matter for health mobility, but intergenerational transmission of health (from slope coefficients) is non-zero for adoptive parent-child pairs, suggesting non-genetic factors are at play in the intergenerational transmission of health.

Results from this study are generally in line with expectations based on previous research. For example, the few studies that have estimated intergenerational health mobility have found $\beta_{1}$ coefficients from equation 1 to be $0.2-0.3$ for self-rated health status using the Panel Study of Income Dynamics (Halliday et al. 2018; Wong et al. 2019), 0.163 for mental health using the 1970 British Birth Cohort Study (Johnston et al. 2013), 0.17 - 0.2 for birth weight in California (Currie \& Moretti 2007), and 0.284 - 0.42 for Body Mass Index using the National Longitudinal 
Survey of Youth and National Health Interview Survey (Classen 2010; Classen \& Thompson 2016).

The analysis in this paper is most similar to that of Halliday et al. (2018) who also use selfreported health status as a health measure, yet results are attenuated from their estimates. There are biases that arise as limitations to this study that relate to this attenuation, specifically a lifecycle bias and attenuation bias that are well documented in intergenerational economic mobility literature (Haider \& Solon 2006; Halliday et al. 2018; Solon 1992). Add Health only observes parent health once, which should predictably increase noise in the parent health estimate due to health shocks that cannot be averaged over time. From figure 4(a) - 4(d) in Halliday et al. (2018), it is clear that had parent health been only observed once, estimates for the rank slope would have been lower in that study. Add Health also has the limitation of observing children earlier in the life course relative to a longer panel dataset such as the PSID. Again from figure 4(g) - 4(h) in Halliday et al. (2018) observing health at later ages, particularly for sons, may increase the relative mobility metric (rank slope). Therefore, it is expected that the estimates of relative mobility in this analysis are attenuated; however, this downward bias should not affect spatial or contextual patterns so long as the bias is similarly present across groups and schools.

Non-Hispanic black parent-child pairs are typically found to experience disadvantages in intergenerational economic mobility (Chetty et al. 2018; Hertz 2005; Mazumder 2014). Early research in intergenerational health mobility, where health is defined by birth weight, finds mixed evidence with respect to relative mobility by race. A dichotomous indicator of low birth weight finds greater intergenerational transmission of health for blacks relative to whites, while a log transformation of continuous birth weight suggests less intergenerational persistence for blacks relative to whites (Currie \& Moretti 2007). Halliday et al. (2018) finds black parent-child pairs may experience higher relative mobility (rank slope coefficient is 0.13 relative to white rank slope coefficient of 0.243), but experience an approximately ten percentile gap in expected absolute health mobility among children whose parents are at the $25^{\text {th }}$ percentile of parent health (see Table 3). That analysis also finds much more downward health mobility for black children whose parents are at the $75^{\text {th }}$ percentile of parent health, a gap of approximately 15 percentiles. Meanwhile, the present analysis only partially corroborates these findings. We find similar estimates of relative mobility for non-Hispanic whites and non-Hispanic blacks as was found in 
Halliday et al. (2018). However, in contrast to that analysis, we find little gap in expected absolute mobility for these groups as shown in Table 3. It is unclear what the source of this discrepancy is - whether it could be due to inclusion of relatively few Hispanic parent-child pairs in the Halliday et al. (2018) analysis, if health discrepancies have not manifested by the relatively early age at which Add Health participants are observed, cohort differences, or if there may be some other reason.

Table 3: Comparison of race stratified results

$$
\text { Add Health }
$$

\begin{tabular}{lcccccc} 
Weighted & All & nH white & nH black & Hispanic & White & Black \\
\hline Rank Slope & 0.174 & 0.201 & 0.154 & 0.0756 & 0.243 & 0.13 \\
E(rank|p25) & 47.24 & 47.05 & 46.97 & 47.5 & 46.5 & 36.8 \\
E(rank|p75) & 55.92 & 57.11 & 54.68 & 51.28 & 58.7 & 43.3 \\
N & 9,771 & 5,637 & 1,913 & 1,611 & 4,555 & 3,139 \\
\hline
\end{tabular}

Source: Authors' calculations using Add Health and estimates from Halliday et al. (2018) Notes: Parent-Child PSID* results from Halliday et al. (2018), which appears to partition the sample by race (not ethnicity). "nH”: non-Hispanic.

Results from this analysis suggest the environment, and not exclusively genetic factors, play a role in determining the intergenerational transmission of health. Specifically, in this analysis relative mobility (the slope coefficient) decreases by approximately 32\% in the adoptee sample relative to the biological parent-child pair sample. Thompson (2014) finds intergenerational elasticity to decrease by approximately 20-30\% for health conditions including asthma, chronic headaches and hay fever and concludes that most of the intergenerational health transmission must be at least in part due to the environment. A more recent study on the intergenerational transmission of Body Mass Index (BMI) however found an adoptee sample with an estimated elasticity of 0.008 , which was not statistically significant, relative to a biological sample with elasticity of 0.202. The authors conclude that, at least in the transmission of BMI, genetics appear to play a relatively large role (Classen \& Thompson 2016). Considering the estimates presented in this paper are concerned with overall health as measured by self-reported health status, it is unsurprising to find slope coefficients attenuated by $32 \%$ in the adoptee sample, between estimates from Thompson (2014) and Classen \& Thompson (2016). Collectively the evidence suggests that while genetic factors certainly play a non-negligible role in the persistence of health across generations, there is a rather sizable portion of intergenerational health mobility which appears to be due to environment factors, which is potentially modifiable. 
Consistent with evidence suggesting the environment may play a role, this analysis also finds parent and child characteristics may matter in a future generation's health. Children without health insurance, whose parents are unmarried, or whose mother has less than a High School education are found to experience lower expected health rank relative to their more advantaged peers. While such stratifications are clearly non-causal, it lends further support to the notion that health is not predetermined, and that policies and the environment can play a modifying role.

Results from school-stratified analyses show there is significant heterogeneity by geography. Some schools have rank slopes that are near zero (suggesting little persistence of health from one generation to the next), while others have rank slopes approaching 0.5, which suggests parent health has a high association with health in the subsequent generation. Similarly, while some places observe children at or above the median of health rank when their parents are observed at the $25^{\text {th }}$ percentile of parent health, other places observe children only achieving the $34^{\text {th }}$ percentile of health rank. Areas identified with high relative health mobility in general also experience high absolute health mobility, although the correlation of these metrics is far from perfect. ${ }^{3}$ These results uncovering high geographic heterogeneity of intergenerational health mobility support research finding high geographic variation in intergenerational economic mobility (Chetty et al. 2014). Halliday et al. (2018) also find health mobility to be lowest in the South, although limitations from PSID prevent a more granular analysis.

A main contribution of this paper is the ability to examine place-based correlates of mobility metrics similar to important work by Chetty et al. (2014) in intergenerational economic mobility. This analysis finds that rural or suburban areas may have lower mobility, while areas with higher portions of minority populations can experience higher relative mobility, but lower absolute mobility among parents in good health. Average socioeconomic status (in this case measured by median income) does not appear to matter much as a correlate of health mobility; however, inequality in income may matter more. This result is consistent with Chetty et al. (2014) results for intergenerational economic mobility. However, while Chetty et al. (2014) find high levels of income dispersion in an area are associated with lower mobility metrics, the analysis here with respect to health mobility suggests the opposite: high inequality may be associated with higher

\footnotetext{
3 The correlation across all sample schools of rank slope mobility and expected absolute upward mobility (at the $25^{\text {th }}$ percentile of parent health) is near $40 \%$.
} 
mobility. One possible rationale for the opposing results may be that exposure to environments with higher socioeconomic status may be related with lower pollution, better schools, and/or better medical facilities. Places with school PTAs and health education requirements may also boost health mobility.

The results of this study rely heavily on self-rated health status as a valid measure of a latent measure of individual health status. To alleviate some concerns with the marginal distribution of a five-point Likert scale, the HALex (Erickson 1998; Erickson et al. 1995; Halliday et al. 2018; Johnson \& Schoeni 2011) is incorporated to better represent marginal differences between selfrated health categories. However, there may be some residual concerns with the measure's performance. Using the advantages afforded by the PSID, Halliday et al. (2018) created an alternative health index which includes 21 indicators of physical and mental health to test the validity of the more simplistic, but widely available, measure of self-rated health. Using their alternative health index, they found results to be consistent with estimates using self-reported health status (Halliday et al. 2018). Additional limitations to the study are the fact that parent health is observed only once, and child health is observed relatively early in the lifecycle. As discussed previously, these factors are expected to attenuate the main national estimates, perhaps even by $20 \%$. However, findings of significant heterogeneity of intergenerational mobility by parent and child characteristics and geography are likely not to be significantly altered under the assumption that such biases operate semi-consistently across space and contexts.

The results of this study suggest that intergenerational health mobility may be relatively high in the United States, but that significant variation exists. Rural and suburban areas tend to experience less mobility, while areas with high income inequality tend to observe higher mobility. Children with health insurance, from homes with married parents, or a mother with more than a High School credential tend to have higher expected health relative to their more disadvantaged peers. Schools with a PTA or a health education requirement may also experience more health mobility. 


\section{References}

Akbulut-Yuksel, M., \& Kugler, A. D. (2016). Intergenerational persistence of health: Do immigrants get healthier as they remain in the US for more generations? Economics \& Human Biology, 23, 136-148.

Autor, D. H., \& Duggan, M. G. (2006). The growth in the social security disability rolls: a fiscal crisis unfolding. Journal of Economic Perspectives, 20(3), 71-96.

Case, A., Fertig, A., \& Paxson, C. (2005). The lasting impact of childhood health and circumstance. Journal of Health Economics, 24(2), 365-389.

Chetty, R., Hendren, N., Jones, M., \& Porter, S. (2018). Race and Economic Opportunity in the United States: An Intergenerational Perspective (No. w24441). Cambridge, MA: National Bureau of Economic Research. https://doi.org/10.3386/w24441

Chetty, R., Hendren, N., Kline, P., \& Saez, E. (2014). Where is the land of opportunity? The geography of intergenerational mobility in the United States. The Quarterly Journal of Economics, 129(4), 1553-1623.

Classen, T. J. (2010). Measures of the intergenerational transmission of body mass index between mothers and their children in the United States, 1981-2004. Economics \& Human Biology, 8(1), 30-43.

Classen, T. J., \& Thompson, O. (2016). Genes and the intergenerational transmission of BMI and obesity. Economics \& Human Biology, 23, 121-133.

Corak, M. (2013). Income Inequality, Equality of Opportunity, and Intergenerational Mobility. Journal of Economic Perspectives, 27(3), 79-102. https://doi.org/10.1257/jep.27.3.79

Currie, J., \& Moretti, E. (2007). Biology as destiny? Short-and long-run determinants of intergenerational transmission of birth weight. Journal of Labor Economics, 25(2), 231264.

DeSalvo, K. B., Bloser, N., Reynolds, K., He, J., \& Muntner, P. (2006). Mortality prediction with a single general self-rated health question. Journal of General Internal Medicine, 21(3), 267.

Erickson, P. (1998). Evaluation of a population-based measure of quality of life: the Health and Activity Limitation Index (HALex). Quality of Life Research, 7(2), 101-114.

Erickson, P., Wilson, R., \& Shannon, I. I. (1995). Years of healthy life.

Haas, S. A., \& Fosse, N. E. (2008). Health and the educational attainment of adolescents: Evidence from the NLSY97. Journal of Health and Social Behavior, 49(2), 178-192.

Haider, S., \& Solon, G. (2006). Life-Cycle Variation in the Association between Current and Lifetime Earnings. The American Economic Review, 21.

Halliday, T., Mazumder, B., \& Wong, A. (2018). Intergenerational Health Mobility in the US. FRB of Chicago Working Paper No. WP-2018-2.

Hertz, T. (2005). Rags, Riches, and Race. Unequal Chances: Family Background and Economic Success, 165.

Hokayem, C., \& Ziliak, J. P. (2014). Health, human capital, and life cycle labor supply. American Economic Review, 104(5), 127-31.

Idler, E. L., \& Angel, R. J. (1990). Self-rated health and mortality in the NHANES-I Epidemiologic Follow-up Study. American Journal of Public Health, 80(4), 446-452.

Johnson, R. C., \& Schoeni, R. F. (2011). The influence of early-life events on human capital, health status, and labor market outcomes over the life course. The BE Journal of Economic Analysis \& Policy, 11(3). 
Johnston, D. W., Schurer, S., \& Shields, M. A. (2013). Exploring the intergenerational persistence of mental health: Evidence from three generations. Journal of Health Economics, 32(6), 1077-1089.

Mazumder, B. (2005). Fortunate Sons: New Estimates of Intergenerational Mobility in the United States Using Social Security Earnings Data. Review of Economics and Statistics, 87(2), 235-255. https://doi.org/10.1162/0034653053970249

Mazumder, B. (2014). Black-white differences in intergenerational economic mobility in the United States. Economic Perspectives, Vol. XXXVIII(No. 1).

McLaughlin, S. J. (2017). Healthy Aging in the Context of Educational Disadvantage: The Role of "Ordinary Magic." Journal of Aging and Health, 29(7), 1214-1234.

Meara, E. R., Richards, S., \& Cutler, D. M. (2008). The gap gets bigger: changes in mortality and life expectancy, by education, 1981-2000. Health Affairs, 27(2), 350-360.

Meyer, B. D., \& Mok, W. K. C. (2018). Disability, earnings, income and consumption. Journal of Public Economics. https://doi.org/10.1016/j.jpubeco.2018.06.011

Solon, G. (1992). Intergenerational Income Mobility in the United States. The American Economic Review, 82(3), 393-408.

Solon, G. (2002). Cross-Country Differences in Intergenerational Earnings Mobility. Journal of Economic Perspectives, 16(3), 59-66. https://doi.org/10.1257/089533002760278712

Solon, G. (2004). A model of intergenerational mobility variation over time and place. Generational Income Mobility in North America and Europe, 38-47.

Thompson, O. (2014). Genetic mechanisms in the intergenerational transmission of health. Journal of Health Economics, 35, 132-146.

Wong, A., Mazumder, B., \& Halliday, T. (2019). The Intergenerational Transmission of Health in the United States: A Latent Variables Analysis. 


\section{Appendix Tables}

Table A1: Intergenerational health mobility stratified by race-ethnicity

\begin{tabular}{lcccccccc} 
& \multicolumn{4}{c}{ IHA Slope } & & \multicolumn{4}{c}{ Rank Slope } \\
& Full & White & Black & Hispanic & Full & White & Black & Hispanic \\
\hline Estimate & 0.086 & 0.100 & 0.075 & 0.052 & 0.174 & 0.201 & 0.154 & 0.076 \\
Standard Error & 0.010 & 0.013 & 0.022 & 0.014 & 0.016 & 0.017 & 0.042 & 0.034 \\
p-value & 0.000 & 0.000 & 0.001 & 0.000 & 0.000 & 0.000 & 0.000 & 0.026 \\
Lower Limit & 0.066 & 0.075 & 0.031 & 0.024 & 0.141 & 0.168 & 0.072 & 0.009 \\
Upper Limit & 0.105 & 0.125 & 0.120 & 0.080 & 0.206 & 0.234 & 0.236 & 0.142 \\
& & & & & & & & \\
& & \multicolumn{2}{c}{ (rank|p25) } & & & $\underline{\text { E(rank|p75) }}$ & \\
& Full & White & Black & Hispanic & Full & White & Black & Hispanic \\
\hline Estimate & 0.472 & 0.471 & 0.470 & 0.475 & 0.559 & 0.571 & 0.547 & 0.513 \\
Standard Error & 0.009 & 0.010 & 0.013 & 0.021 & 0.007 & 0.008 & 0.018 & 0.020 \\
p-value & 0.000 & 0.000 & 0.000 & 0.000 & 0.000 & 0.000 & 0.000 & 0.000 \\
Lower Limit & 0.455 & 0.452 & 0.443 & 0.434 & 0.545 & 0.556 & 0.512 & 0.473 \\
Upper Limit & 0.489 & 0.489 & 0.496 & 0.516 & 0.574 & 0.586 & 0.581 & 0.552 \\
\hline
\end{tabular}

Source: Authors' calculations using Add Health

Notes: Table A1 corresponds to Figure 1 above. IHA slope is the Intergenerational Health Association slope ( $\beta_{1}$ coefficient from equation 1 ) using HALex level self-rated health in both generations. Rank slope is the $\beta_{1}$ coefficient using health ranks in both generations. $\mathrm{E}(\operatorname{rank} \mid \mathrm{p} 25)$ is the expected health rank for a child conditional on his/her parent observed at the $25^{\text {th }}$ percentile of parent health, and $\mathrm{E}(\mathrm{rank} / \mathrm{p} 75)$ is the expected health rank for children whose parent(s) are observed at the $75^{\text {th }}$ percentile of parent health. Estimates are weighted, and use the full Add Health sample. Lower and Upper Limits refer to the 95\% confidence intervals.

Table A2: Distribution of school-stratified estimates of intergenerational health mobility

\begin{tabular}{lcccc} 
& IHA Slope & Rank Slope & E(rank|p25) & E(rank|p75) \\
\hline Minimum & -0.245 & -0.331 & 23.0 & 6.4 \\
$10^{\text {th }}$ & -0.018 & -0.020 & 34.5 & 43.3 \\
$25^{\text {th }}$ & 0.020 & 0.041 & 41.1 & 49.4 \\
$50^{\text {th }}$ & 0.084 & 0.164 & 47.0 & 55.2 \\
$75^{\text {th }}$ & 0.151 & 0.249 & 53.1 & 60.7 \\
$90^{\text {th }}$ & 0.212 & 0.338 & 58.6 & 64.3 \\
Maximum & 0.388 & 0.490 & 75.8 & 77.5 \\
\hline
\end{tabular}

Source: Authors' calculations using Add Health

Notes: Table A2 corresponds to Figure 2 above. IHA slope is the Intergenerational Health Association slope ( $\beta_{1}$ coefficient from equation 1 ) using HALex level self-rated health in both generations. Rank slope is the $\beta_{1}$ coefficient using health ranks in both generations. $\mathrm{E}(\operatorname{rank} \mid \mathrm{p} 25)$ is the expected health rank for a child conditional on his/her parent observed at the $25^{\text {th }}$ percentile of parent health, and $\mathrm{E}$ (rank $\mid \mathrm{p} 75$ ) is the expected health rank for children whose parent(s) are observed at the $75^{\text {th }}$ percentile of parent health. Estimates are not weighted, but use only children identified in the core sample to produce school-specific results. 